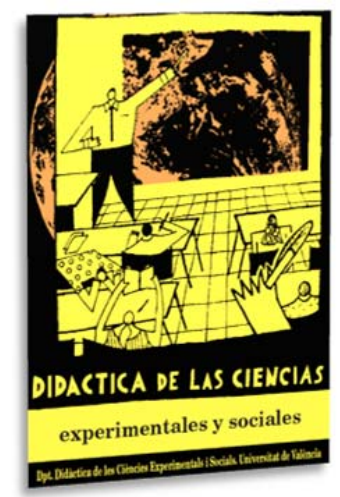

\title{
El iPad en la Educación científica de estudiantes de Secundaria y Bachillerato
}

\section{The iPad in scientific Education of students in Secondary Education and High School}

DOI: $10.7203 / D C E S .36 .12661$

\author{
Ana Ruiz Santiago \\ Universidad Complutense de Madrid, aruiz07@ucm.es
}

Alberto Muñoz Muñoz

Universidad Complutense de Madrid, albemuno@ucm.es

ORCID iD: http://orcid.org/0000-0002-1152-7444

$\mathbf{M}^{\mathrm{a}}$ Dolores Valladolid Carriazo

Colegio Hélicon, lvalladolid@colegiohelicon.com

Juan Peña Martínez

Universidad Complutense de Madrid, juan.pena@quim.ucm.es

ORCID iD: http://orcid.org/0000-0001-9395-7976

\begin{abstract}
RESUMEN: Las nuevas tecnologías han calado muy profundamente en Educación. Hemos analizado las percepciones de estudiantes y docentes sobre el uso de la tablet como herramienta para enseñar ciencias, en un centro de ESO./Bachillerato donde cada estudiante dispone de un iPad. Estudiantes y docentes consideran que el mayor rendimiento del iPad se obtiene en asignaturas relacionadas con las ciencias de la vida. Sin embargo, la mayoría de los docentes reconoce que no aprovecha todos los recursos que ofrece el iPad. Discutimos cómo la tecnología no garantiza una mejora en la calidad de la educación científica si no va acompañada de una adecuada formación del profesorado y de unas estrategias didácticas que faciliten su correcta implementación en las aulas.
\end{abstract}

Palabras Clave: Educación Secundaria Obligatoria, Educación científica, iPad, Nuevas tecnologías, tablet.

ABSTRACT: The new technologies have penetrated deeply into Education. We have analyzed the perception of students and teachers on the use of tablets as teaching sciences tools, in a Secondary and High School where each student is provided with an iPad. Both students and teachers consider that the highest performance of the iPad is achieved in subjects related to life sciences. However, most teachers recognize that they do not take advantage of all the iPad resources. We discuss why technology does not guarantee an improvement in the quality of scientific education without an adequate training of teachers and some didactic strategies to implement its suitable use in the classrooms.

KEYwORDS: Secondary Compulsory Education, Scientific education, iPad, New technologies, tablet.

Fecha de recepción: junio de 2018

Fecha de aceptación: febrero de 2019

Agradecemos al equipo directivo y el profesorado del Colegio Hélicon la predisposición y facilidades para llevar a cabo este estudio. 


\section{INTRODUCCIÓN}

\subsection{Las nuevas tecnologías y la enseñanza de las ciencias}

El vertiginoso desarrollo de las denominadas tecnologías de la información y la comunicación (TIC) en los últimos años las ha convertido en un elemento que ha calado profundamente en la sociedad a diferentes niveles. En el contexto de la enseñanza-aprendizaje, las TIC se han convertido en pocos años en una herramienta muy destacada. Tal ha sido su influencia a nivel mundial que la calidad de la educación de los países tecnológicamente desarrollados parece residir en la capacidad de la escuela para reducir la creciente brecha digital, es decir, la división social entre quienes saben y no saben hacer uso de las nuevas tecnologías (García-Vera, 2004; Aunión, 2009). En este sentido, muchos países han puesto en marcha actuaciones con distintos niveles de compromiso para tratar de cubrir esa brecha.

Son cada vez más los centros educativos que apuestan por los denominados programas 1:1, un ordenador por cada estudiante. Estos programas tienen como objetivo impulsar una renovación metodológica en la enseñanza, desarrollando la Competencia Digital entre sus alumnos. Esta competencia, establecida en el currículo de Educación Primaria, E.S.O. y Bachillerato como una de las 7 competencias básicas, está fundamentada en el uso creativo, crítico y seguro de las TIC. Los programas 1:1 comenzaron a proliferar a comienzos del siglo XXI, coincidiendo con el desarrollo de dispositivos informáticos portátiles de bajo coste y la creciente disponibilidad de conectividad inalámbrica en los centros educativos. Estos programas proporcionan acceso a un dispositivo TIC, conectado a internet a través de la red escolar y a diversos softwares educativos. Su objetivo es ofrecer a cada estudiante acceso individualizado a las TIC de manera permanente y ubicua, a través de un dispositivo personal como un portátil, una tablet, etc. (González, 2011).

La enseñanza de las ciencias es un campo que puede obtener un interesante provecho de la reciente revolución tecnológica y su dimensión en las aulas. La alfabetización tecno-científica es fundamental para dotar a los estudiantes de modelos explicativos para interpretar el mundo y enfrentar los retos del siglo XXI (Gómez-Crespo, Pozo y Gutiérrez-Julián, 2004; Gordillo, Tedesco, López, Acevedo, Echeverria y Osorio, 2009). Un recurso empleado tradicionalmente en la enseñanza de las ciencias son las prácticas de laboratorio, que permiten cuestionar las ideas alternativas de los estudiantes, formuladas como hipótesis previas a los experimentos, así como encontrar sentido a las ideas científicas cuando son aplicadas para explicar fenómenos observables (Ariza y Quesada, 2014). La enseñanza de las ciencias está sufriendo actualmente una transformación metodológica tal, que ya no es necesario disponer de un laboratorio físicamente, sino que basta con un simple 'click' de ratón para acceder a un enorme abanico de herramientas que nos ofrecen las TIC, como laboratorios virtuales, simuladores, herramientas de modelización, adquisición, representación y ordenación de datos o herramientas de trabajo cooperativo. En este nuevo contexto, es coherente que las competencias científica y tecnológica se encuentren incluidas en la misma categoría: “Competencia matemática y competencias básicas en ciencia y tecnología”, puesto que ambas contribuyen conjuntamente al desarrollo del pensamiento científico.

\subsection{Las tablets en la enseñanza de las ciencias}

Desde el punto de vista de la enseñanza de las ciencias, las tablets ofrecen mucho más que la simple posibilidad de navegar por Internet. Hoy en día ni siquiera es necesario disponer de una red a la que conectarse para poder desarrollar trabajos que permitan trabajar la competencia científica; esto es debido a que esta herramienta ofrece un sinfín de recursos con aplicación científica, como aplicaciones de cartografía y GPS, radares, o como sensores como vibrómetros, sonómetros, etc. Las tablets pueden permitir a los estudiantes llegar a entender las dimensiones del espacio mediante simulaciones del Universo, algo muy dificultoso en la enseñanza tradicional en el aula (Nieves, 
2013). Con todo esto resulta obvio que las tablets deben de, al menos, fomentar la curiosidad, que es un primer paso para trabajar la competencia científica.

Además de su uso para la experimentación y el potencial de las aplicaciones multimedia interactivas, las tablets también son una herramienta muy útil a la hora de documentarse sobre cualquier tema, gracias a las aplicaciones de consultas de obras, libros y artículos y a las de gestión de la información (Pedrajas, 2005; García y Rey, 2012). Al mismo tiempo, con las TIC se fomenta el desarrollo de determinadas destrezas intelectuales como el autoaprendizaje, la capacidad integradora (Lowy, 1999) y el trabajo cooperativo (Guitert, Romeu y Pérez-Mateo, 2007). La mayoría de autores coinciden en señalar como grandes ventajas por un lado, la rapidez de los sistemas operativos y, por otro, la posibilidad de integrar los contenidos en unidades específicas orientadas según niveles educativos o temáticas curriculares determinadas. En este sentido, destaca que el reemplazo del significativo número de libros de textos que los estudiantes deben cargar cotidianamente en sus mochilas por la tablet, podría contribuir efectivamente en los aspectos preventivos de su salud física (Marés, 2012).

Uno de los aspectos que favorecen la inclusión de tablets en los centros educativos es que el proceso de enseñanza-aprendizaje se vuelve más individualizado. Sin embargo, hay que diferenciar entre educación individualizada y educación personalizada. En este tipo de metodología quien lleva las riendas del aprendizaje no es el estudiante, sino la aplicación "inteligente” de la tablet, de manera que tanto estudiante como docente pueden pasar a ocupar lugares secundarios. En este extremo de la balanza un número cada vez mayor de profesionales y familiares de alumnos muestra cierto recelo a la hora de aceptar de buen grado este tipo de innovaciones. Esto se debe, en la mayor parte de las ocasiones, al desconocimiento de los efectos resultantes del empleo de estas tecnologías dentro del aula. Padres y madres generalmente están de acuerdo en la idea de que actualmente las nuevas tecnologías son indispensables en educación, pero curiosamente también se encuentran entre los principales detractores de las tablets ya que, a pesar de manejar en su vida diaria dispositivos similares, se ven impotentes para ejercer un control con garantías en sus hijos, delegando dicha responsabilidad en el centro educativo (Martínez, 2015). De hecho, existen estudios que advierten de los impactos negativos que conllevan como la incapacidad para filtrar información relevante, hiperactividad, apatía, adicción, la deshumanización del aprendizaje, el mal funcionamiento de la memoria de trabajo etc., lo que puede implicar importantes pérdidas de atención y concentración (Gracia, Vigo, Marcó y Fernández, 2002; Blanch, 2013).

\subsection{Calidad educativa y nuevas tecnologías}

Si bien las nuevas tecnologías abren un abanico muy interesante de posibilidades en el contexto de la enseñanza-aprendizaje, su vertiginosa implantación en contextos educativos puede generar problemas si se considera como un fin en sí mismo y no como una herramienta que mejore la calidad de la educación. La disponibilidad y acceso a las nuevas tecnologías en centros educativos no es, o no debe ser, una bandera de elevada calidad educativa, ya que esta última depende críticamente del uso y el rendimiento didáctico que se obtenga de los dispositivos (Ancira y Gutiérrez, 2011). Por ejemplo, algunos estudios muestran que muchos docentes españoles de Educación Primaria y Secundaria no son suficientemente competentes en el uso de las TIC (Sigales, Mominó, Meneses y García, 2008), por lo que se ha sugerido que la formación del profesorado es el primer obstáculo a superar, haciéndose necesaria una formación continua y una actualización didáctica que responda a las necesidades del profesorado (Palmero y Rodríguez, 2012). Esta es una etapa clave para una verdadera revolución en la educación científica, y depende críticamente de la predisposición de los docentes a incorporar las nuevas tecnologías en sus clases y de una verdadera voluntad de formación continua (Martínez, 2015), ya que la evolución de las TIC está en constante desarrollo (Marcelo y Estebaranz, 1999). Una formación deficiente en un aspecto que la mayoría de los alumnos dominan sin problemas, como "nativos digitales", puede ser incluso sinónimo de pérdida de autoridad (Aunión, 2009; Tallone, 2011). 
La comunidad educativa se encuentra dividida en cuanto al uso de las TIC, existiendo dos corrientes predominantes. Por un lado, la de aquellos que apoyan a ultranza la inserción de las nuevas tecnologías al ámbito escolar, promoviendo el desplazamiento de los métodos más tradicionales (Vásquez-Rodríguez, 2015). Por otro lado, la visión de aquellos que se muestran cautelosos y reticentes, denunciando una serie de carencias y problemas derivados de las nuevas tecnologías como consecuencia de las pocas investigaciones publicadas al respecto (Gil, 2013). La dinámica global empuja a las escuelas y profesores a adaptarse de manera acelerada a las nuevas tecnologías, pero muchas veces se enfrentan a las reticencias de padres y madres que no llegan a confiar del todo en ellas. Esto no hace más que acrecentar el desconcierto entre los estudiantes, que está condicionado por el hecho de que la sociedad actual está aún influenciada por los modelos enseñanza-aprendizaje tradicionales (Gil, 2013; Martínez 2015). Por todo ello, son imprescindibles estudios que evalúen el uso e impacto de la implantación de las nuevas tecnologías en la calidad educativa y el rendimiento escolar, que a su vez ayuden a promover un correcto uso para aumentar su potencial didáctico (Carneiro, Toscano y Díaz, 2009). El presente estudio es una contribución en este sentido, ya que analiza las percepciones de estudiantes y docentes de un centro educativo que recientemente ha implementado un programa 1:1 en todos los niveles de la Educación Secundaria Obligatoria y en el primer curso de Bachillerato, donde cada alumno posee como herramienta principal de trabajo un iPad4.

\subsection{Objetivos}

El objetivo general del presente trabajo es analizar el papel del iPad en la enseñanzaaprendizaje de las ciencias, en base a las visiones de estudiantes y docentes de Educación Secundaria Obligatoria y Bachillerato. En concreto, nos planteamos los siguientes objetivos específicos:

1.- Analizar cómo afecta el uso del iPad al desarrollo del pensamiento científico y a la competencia científica por parte del alumnado y del profesorado.

2.- Determinar las asignaturas de ciencias en las que más se desarrolla la competencia digital de estudiantes y docentes.

3.- Examinar en qué grado el iPad fomenta la autonomía, la cooperación y la creatividad de estudiantes y docentes.

4.- Determinar los efectos del iPad en la atención y concentración del alumnado.

5.- Comparar la percepción de estudiantes y docentes de diferentes cursos de la Enseñanza Secundaria Obligatoria y el Bachillerato sobre los beneficios pedagógicos del iPad.

6.- Realizar una serie de propuestas para compensar las desigualdades sobre los recursos tecnológicos, con la intención de mejorar la rentabilidad de los recursos empleados en la metodología 1:1.

\section{MetodologíA}

\subsection{Contexto y población de estudio}

El estudio se ha llevado a cabo en el mes de abril del curso académico 2015-2016, en un centro educativo de la Comunidad de Madrid. El centro cuenta con 1300 alumnos desde primer ciclo de Educación Infantil hasta Bachillerato, siendo privado para estas dos etapas, y en régimen de concierto educativo en segundo ciclo de Educación Infantil, Primaria y Educación Secundaria Obligatoria. El centro ha llevado a cabo recientemente una profunda reforma, desarrollando un plan de innovación metodológica que se inició a comienzos del curso académico 2014-2015 y cuyos objetivos son fomentar la autonomía del alumnado y el desarrollo del espíritu cooperativo y emprendedor, así como la motivación a partir del trabajo por proyectos, la resolución de problemas y el trabajo de las inteligencias múltiples. Como parte de esta transformación, en la etapa de 
Educación Secundaria Obligatoria el libro de texto ha sido sustituido por un iPad facilitado por el centro como herramienta principal de estudio y trabajo, tanto para estudiantes como para docentes. En los iPads los alumnos cuentan con libros de texto digitales y una plataforma virtual de aprendizaje (Xtend). También cuentan con varios sistemas para la comprobación de uso del dispositivo por parte del estudiante, como la plataforma para padres y un sistema (MDM) de control de tablets. Los estudiantes no sólo emplean el iPad en las clases teóricas de diferentes asignaturas, sino que también se ayudan de él a la hora de realizar los talleres prácticos con el fin de responder las preguntas que les van surgiendo.

En este estudio han participado todos los estudiantes de Educación Secundaria Obligatoria (E.S.O.) y del primer curso de Bachillerato del centro, así como todos los docentes que imparten alguna asignatura científica en cualquiera de estas etapas. La muestra comprendió un total de 307 estudiantes y 12 docentes de ciencias. La distribución de estudiantes entre los diferentes cursos fue la siguiente: 3 grupos de $1^{\circ}$ de E.S.O. ( $\mathrm{n}=68$ estudiantes), 3 grupos de $2^{\circ}$ de E.S.O. $(\mathrm{n}=78$ estudiantes), 4 grupos de $3^{\circ}$ de E.S.O. ( $n=74$ estudiantes), 3 grupos de $4^{\circ}$ de E.S.O. ( $n=64$ estudiantes) y 1 grupo de $1^{\circ}$ de Bachillerato $(n=23$ estudiantes). Los alumnos del segundo curso de Bachillerato no pudieron participar en este estudio debido a que no usan iPad, porque la implantación del dispositivo comenzó en el curso 2014-2015 únicamente para la etapa de E.S.O. Los 12 docentes que participaron en el estudio impartieron las asignaturas de Matemáticas (11 docentes), Física y Química (6 docentes), Tecnología (3 docentes), Ciencias Naturales (3 docentes), Biología y Geología (4 docentes), Biología (1 docente) y Anatomía (1 docente).

\subsection{Diseño del estudio}

La primera fase de la investigación consistió en la elaboración de varios cuestionarios dirigidos a los estudiantes y docentes de ciencias que participaron en el estudio. Los cuestionarios, aunque partían de una base común, fueron diseñados de manera diferente para estudiantes y docentes con el fin de ajustarlos a las características de cada colectivo, apoyándose en los cuestionarios realizados por Galaz y Weil (2014). Para la elaboración y validación de los cuestionarios se contó con la colaboración de diversos docentes. Los cuestionarios presentan una estructura cerrada, con posibilidad de realizar alguna observación concreta en determinadas preguntas, constan de 16 preguntas para los alumnos y 17 para los profesores (ANEXO I). Los aspectos principales en los que se han centrado ambos cuestionarios son los siguientes:

- Relación del uso del iPad con las asignaturas científicas impartidas.

- Uso del dispositivo dentro y fuera de clase tanto por parte del alumnado como del profesorado.

- Recursos empleados y métodos evaluación relacionados con el iPad. En este apartado cabe aclarar en qué consiste cada uno de los métodos de evaluación planteados: Socrative, un cuestionario tradicional en versión virtual, Kahoot, un juego interactivo donde hay un ranking de alumnos según el tiempo que tarden en contestar correctamente a preguntas sobre un tema concreto, y el típico examen en papel.

- Relación entre el desarrollo del pensamiento científico y el uso del iPad.

- Beneficios del iPad en cuanto en el proceso de enseñanza-aprendizaje científico.

Los cuestionarios de los estudiantes fueron completados en horario de clases durante alguna de las asignaturas relacionadas con los mismos, y los cuestionarios de los docentes fueron realizados en horario lectivo, pero fuera de las aulas. Los datos se analizaron con el programa Statistica 7.1 mediante Análisis de la Varianza y test LSD de comparaciones a posteriori. 


\section{Resultados}

\subsection{Perspectiva del alumnado}

Las herramientas preferidas por los estudiantes para estudiar son el iPad y los apuntes, mientras que el tradicional libro físico y el ordenador quedan relegados a la segunda y tercera posición, notablemente por debajo de los dos primeros. Este patrón se mantiene a lo largo de todos los cursos de ESO, pero es diferente en $1^{\circ}$ de Bachillerato, donde los apuntes y el libro físico son preferidos al iPad y al ordenador (Gráfico 1).

GRÁFICO 1. Herramientas preferidas para estudiar en alumnos de E.S.O. y Bachillerato

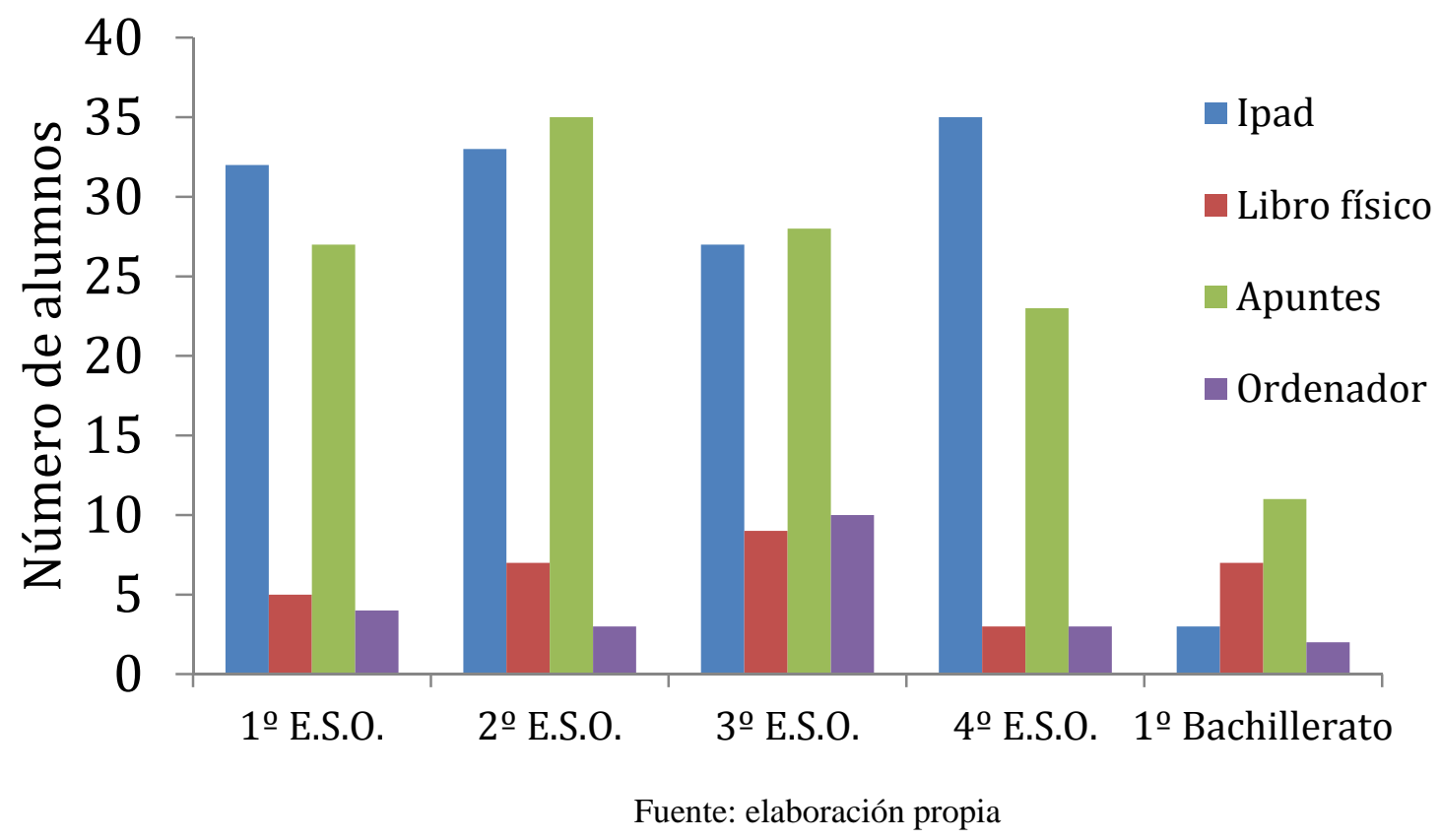

El 83,4\% de los alumnos afirma que fuera de clase usa el iPad bastante o mucho para estudiar y el 90,8\% de los alumnos afirman no usarlo o usarlo poco para jugar. Los alumnos consideran que las asignaturas que más permiten trabajar con el iPad son las relacionadas con las ciencias de la vida, es decir, “Biología y Geología”, “Ciencias Naturales” y "Biología y Anatomía”. En el otro extremo de la balanza se encontrarían las Matemáticas, donde el iPad se usaría poco o nada según la mayoría de los alumnos. En un punto intermedio quedarían "Física y Química” y "Tecnología”, donde el uso del iPad muestra un patrón menos uniforme a lo largo de los cursos. Este patrón es general en todos los cursos de E.S.O., observándose una disminución en el uso del iPad en $1^{\circ}$ de Bachillerato (gráfico 2). 
GRÁFICO 2. Uso del iPad en differentes asignaturas de ciencias en E.S.O. y Bachillerato
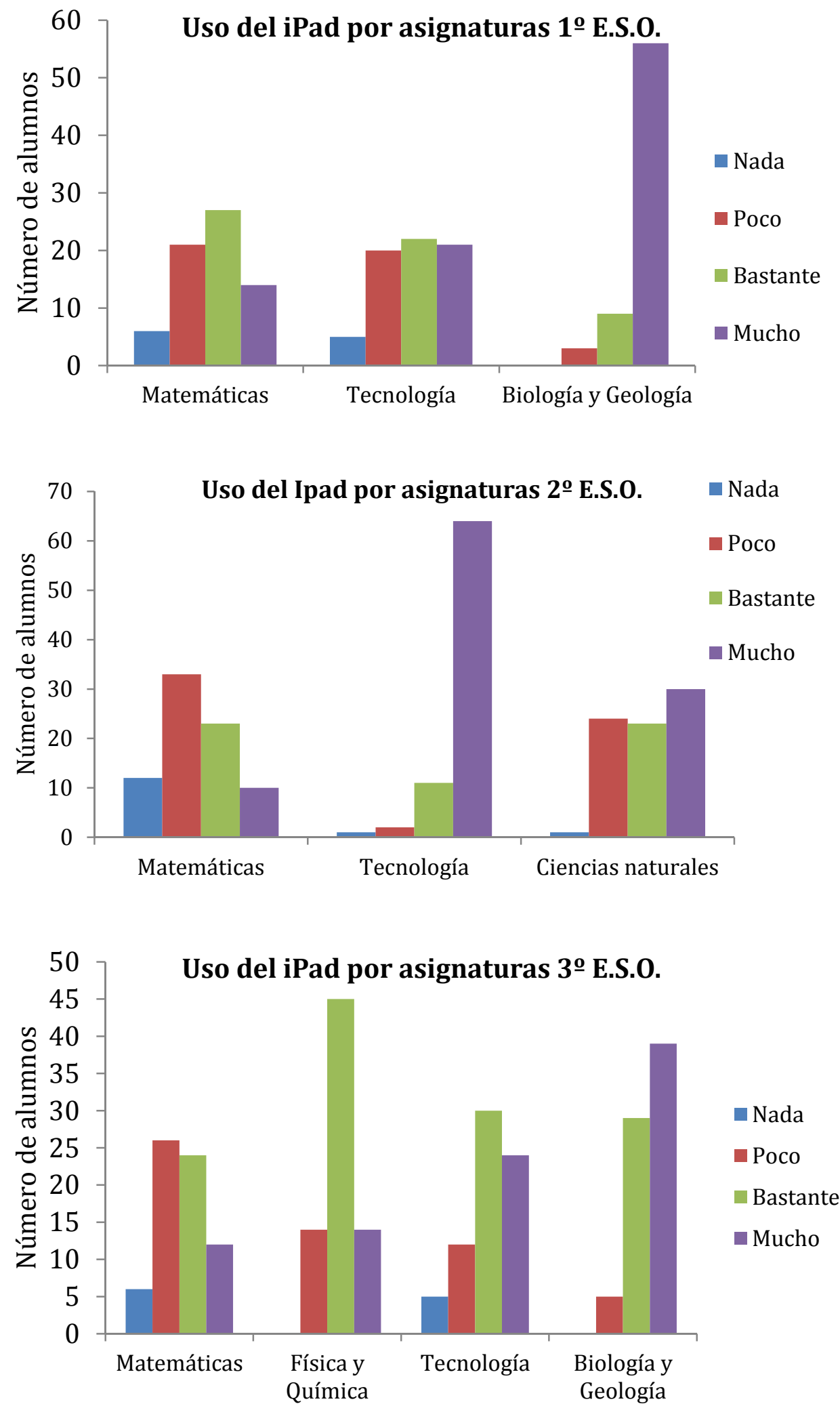

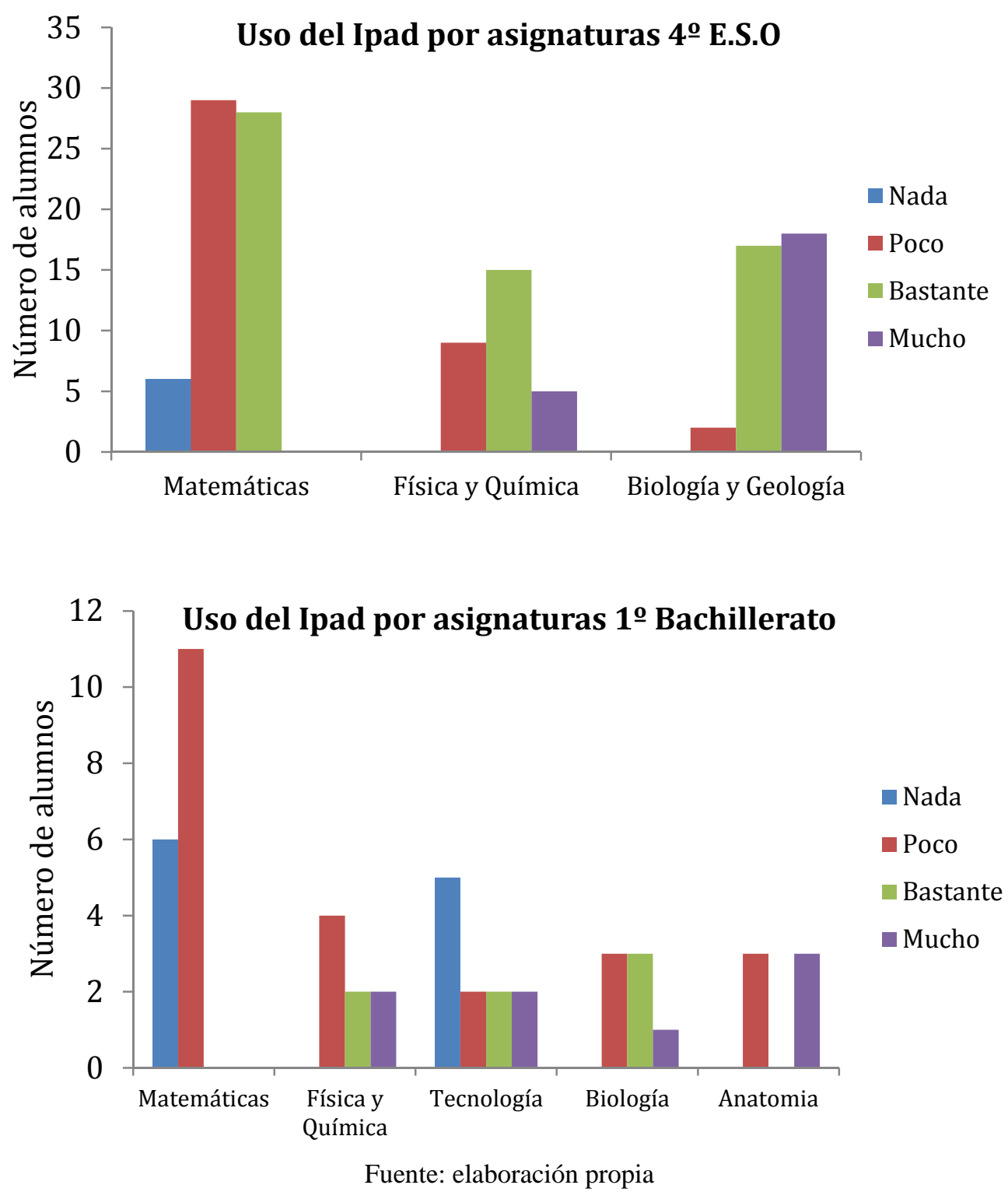

Respecto a la utilidad del iPad en diferentes tareas relacionadas con el proceso de enseñanza aprendizaje, en la Tabla 1 se puede observar que los estudiantes consideran que el iPad es muy útil para la búsqueda y agrupamiento de la información, así como para conseguir elaboraciones y presentaciones de trabajos más estéticas. Además, consideran que el iPad fomenta en alto grado su creatividad a la hora de realizar los trabajos académicos y que es una buena herramienta para fomentar el trabajo cooperativo, aunque algo menos eficaz en el fomento de la autonomía (Tabla 1). Contrastan estos resultados con su escasa utilidad para simulaciones y laboratorios virtuales y para el análisis de datos y la representación gráfica. 
TABLA 1. Utilidad del iPad para diferentes tareas académicas según estudiantes de Educación Secundaria Obligatoria y Bachillerato

\begin{tabular}{lcccc}
\hline & NADA & POCO & BASTANTE & MUCHO \\
\hline Búsqueda de información & $3 \%$ & $12 \%$ & $\mathbf{3 7 \%}$ & $\mathbf{4 8 \%}$ \\
\hline Orden y agrupamiento de información & $4 \%$ & $16 \%$ & $\mathbf{4 6 \%}$ & $\mathbf{3 4 \%}$ \\
\hline Análisis y representación gráfica & $\mathbf{6 5 \%}$ & $\mathbf{2 7 \%}$ & $5 \%$ & $3 \%$ \\
\hline Fomento trabajo cooperativo & $1 \%$ & $4 \%$ & $\mathbf{2 9 \%}$ & $\mathbf{6 6 \%}$ \\
\hline Desarrollo de la creatividad y estética & $2 \%$ & $8 \%$ & $\mathbf{2 8 \%}$ & $\mathbf{6 2 \%}$ \\
\hline Fomento trabajo autónomo & $15 \%$ & $32 \%$ & $36 \%$ & $17 \%$ \\
\hline Simulaciones & $\mathbf{4 3 \%}$ & $\mathbf{4 4 \%}$ & $12 \%$ & $1 \%$ \\
\hline Laboratorios virtuales & $\mathbf{9 0 \%}$ & $\mathbf{9 \%}$ & $1 \%$ & $0 \%$ \\
\hline
\end{tabular}

Fuente: elaboración propia

Respecto a los efectos del iPad en la distracción del alumnado, llama la atención que se percibe un incremento con el curso. En primero de E.S.O. algo más del $20 \%$ de los estudiantes consideran que iPad es un elemento que distrae bastante o mucho, mientras que en bachillerato ese porcentaje sube hasta casi el 70\% (Gráfico 3).

GRÁFICO 3. Fomento de la distracción por el iPad y sistemas de evaluación preferidos según los alumnos de E.S.O. y Bachillerato
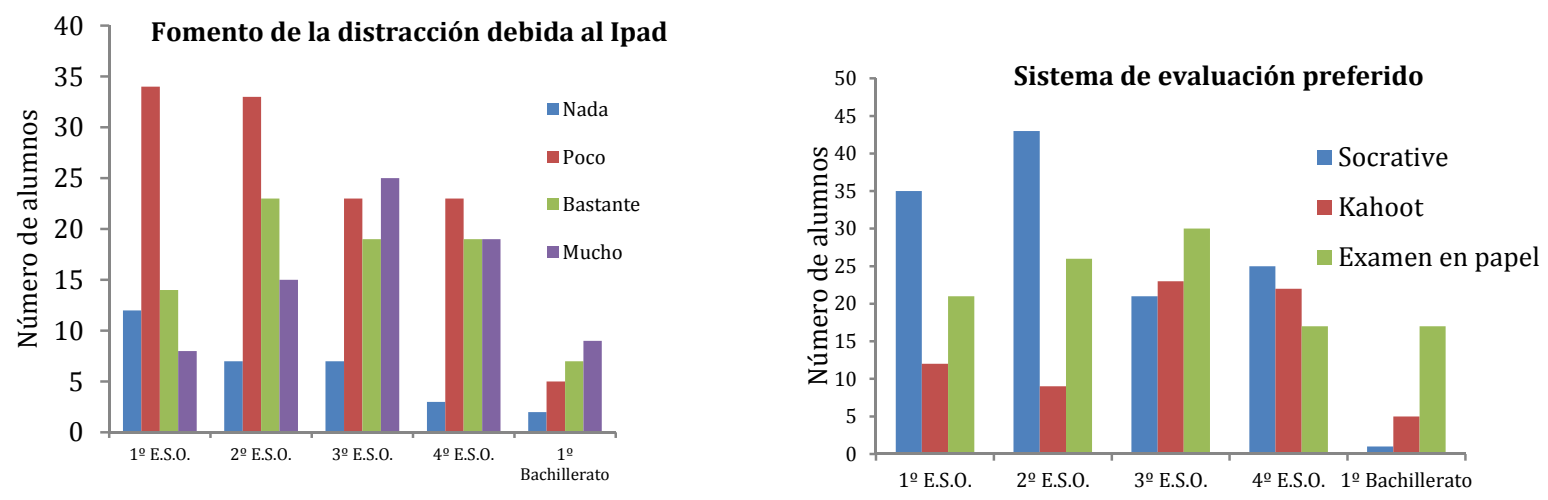

Fuente: elaboración propia

Se observa una evolución similar (en detrimento del iPad) respecto al sistema de evaluación preferido por los estudiantes. En primero y segundo de E.S.O. prefieren el Socrative (cuestionario online), quedando el examen en papel en segundo lugar, mientras que los estudiantes de bachillerato prefieren el tradicional examen en papel (Gráfico 3). Se aprecian diferencias significativas entre cursos en la calificación global del iPad por parte de los estudiantes (ANOVA: $\mathrm{F}_{4,302}=3.15, \mathrm{P}=0.014$ ), de manera que hay un patrón claro por el que la calificación media se va reduciendo a medida que progresamos en los cursos (Gráfico 4). 
GRÁFICO 4. Nota media de los beneficios del iPad en el aprendizaje según los alumnos de diferentes cursos y los profesores de ciencias (las líneas sobre barras indican las desviaciones estándar)

$$
8
$$

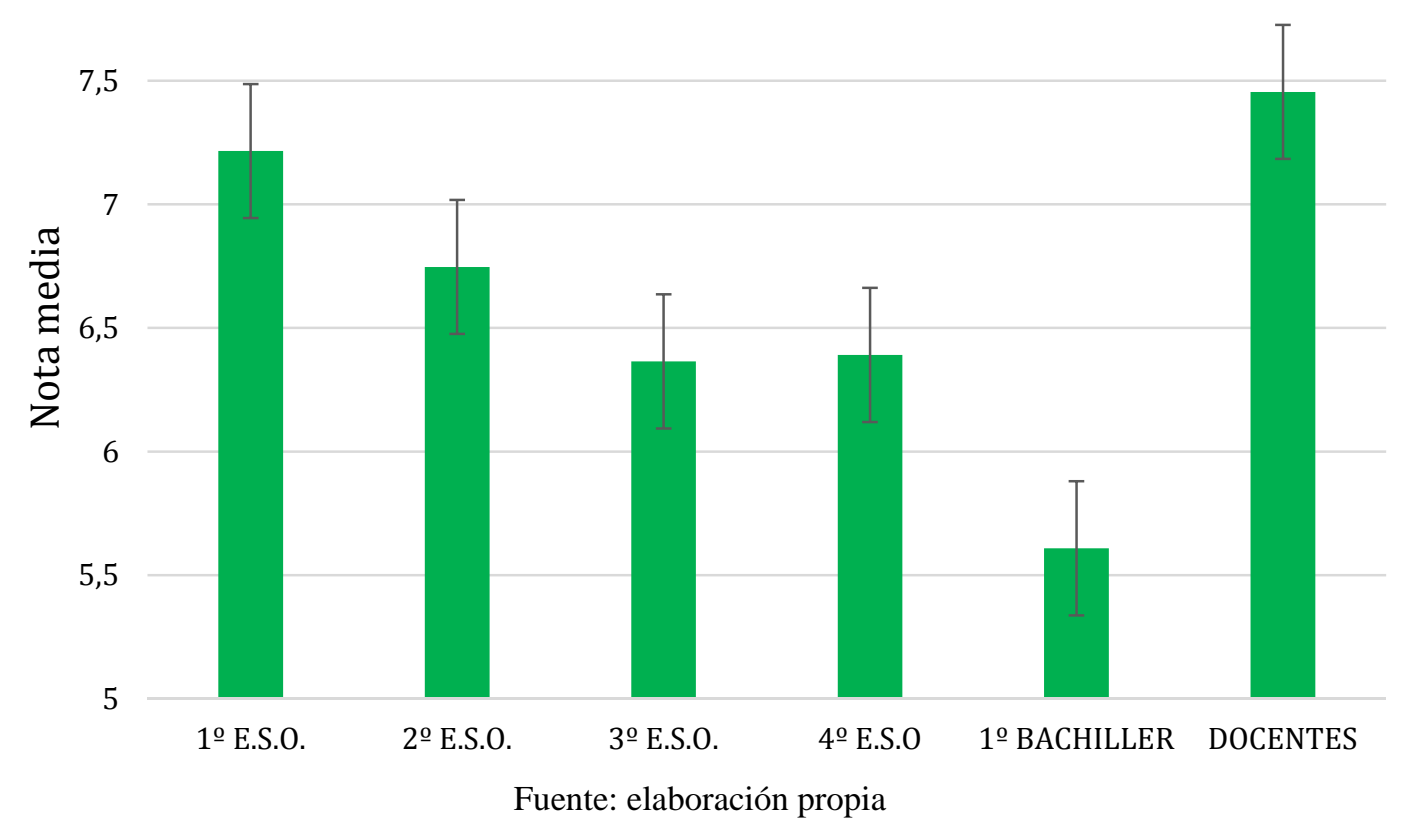

\subsection{Perspectiva del profesorado}

$\mathrm{Al}$ igual que los estudiantes, los docentes también consideran que las asignaturas relacionadas con las ciencias de la vida (Biología y Geología, Ciencias Naturales, Biología y Anatomía) es donde se obtiene un mayor rendimiento del iPad. Todos los profesores que impartían este tipo de asignaturas afirmaron que usan mucho el iPad (Gráfico 5).

GRÁFICO 5. Utilidad del iPad por parte del profesorado según las asignaturas impartidas

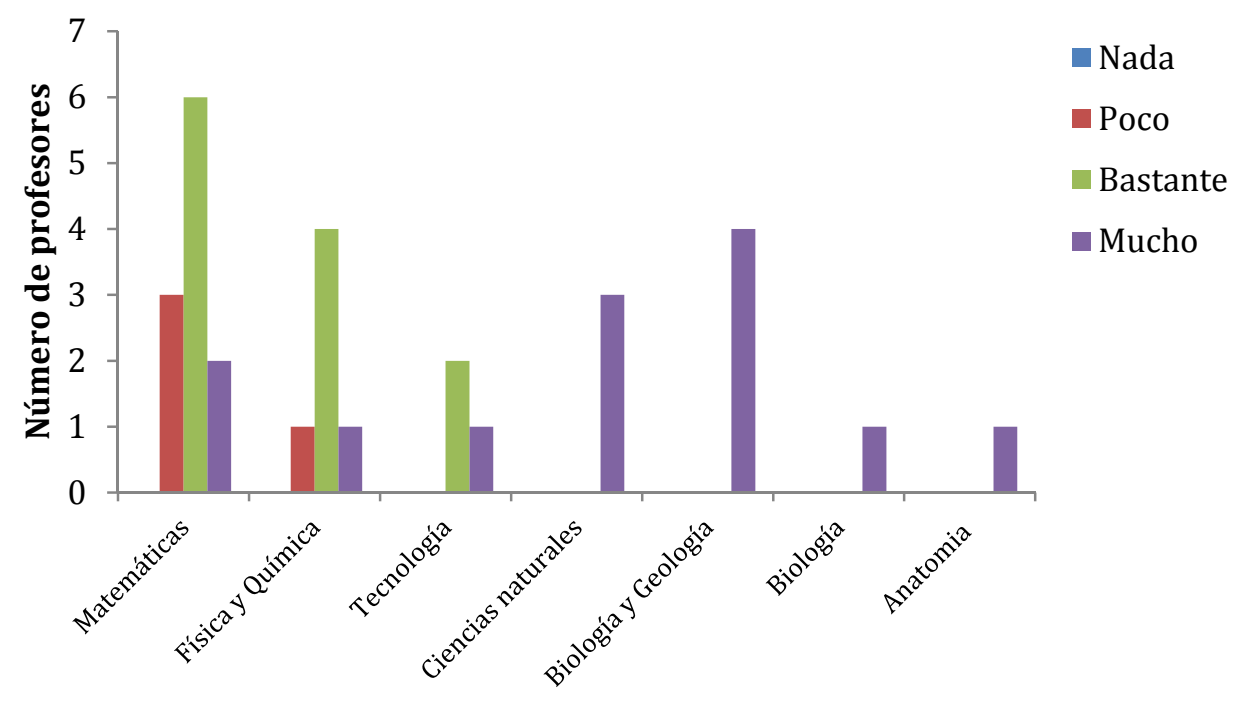

Fuente: elaboración propia 
La elaboración de apuntes y a la búsqueda en Internet desde clase son las actividades que representan un mayor uso del iPad para los docentes, en contraste con la elaboración de videos y juegos son las actividades menos desarrolladas a través del iPad (Gráfico 6).

GRÁFICO 6. Uso del iPad según distintas actividades por parte del profesorado

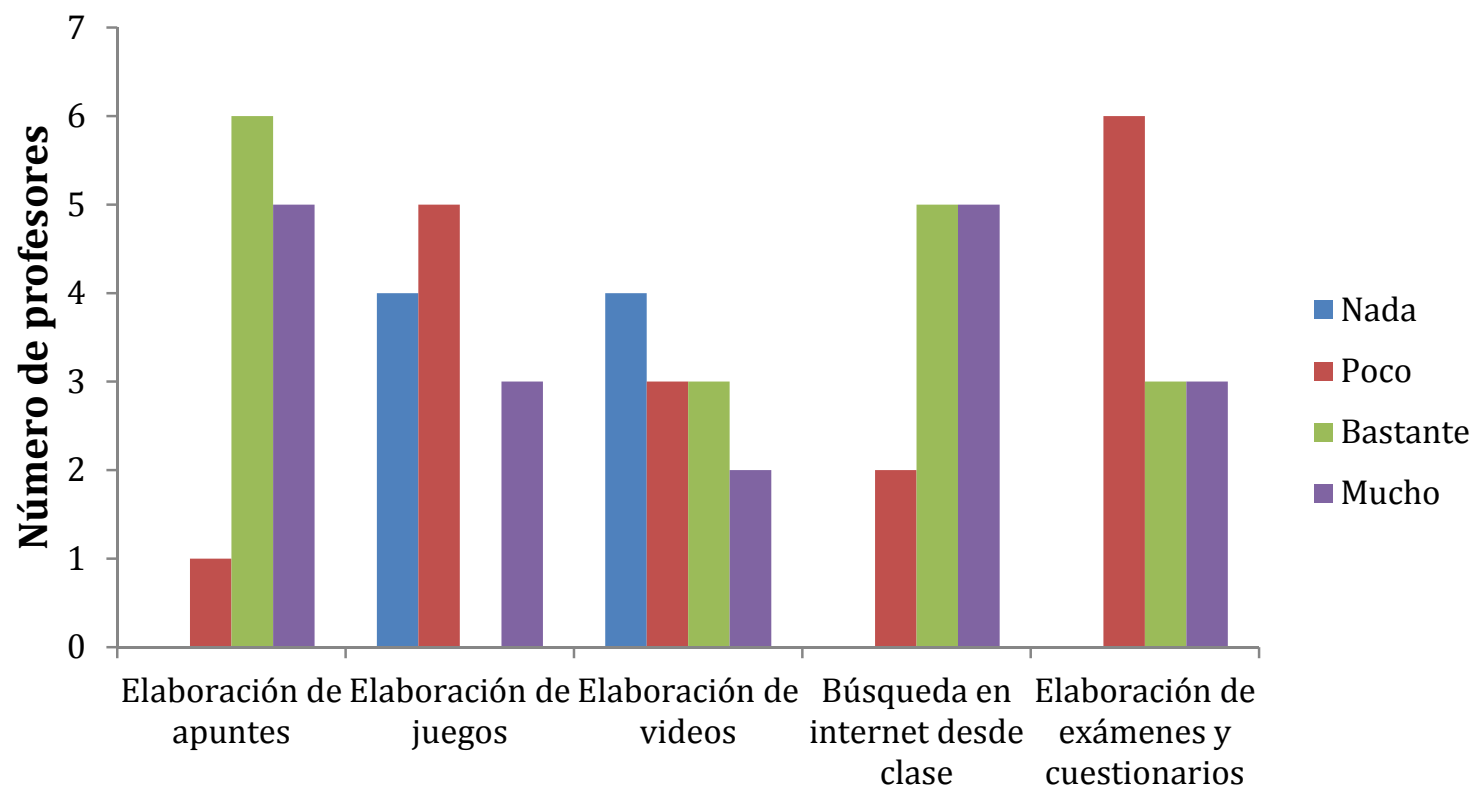

Fuente: elaboración propia

TABLA 2. Contribución del iPad en el desarrollo de diferentes actividades según el profesorado

\begin{tabular}{lcccc}
\hline & NADA & POCO & BASTANTE & MUCHO \\
\hline Indagar y documentarse & $0 \%$ & $0 \%$ & $\mathbf{2 5 \%}$ & $\mathbf{7 5 \%}$ \\
\hline Agrupar y ordenar información & $0 \%$ & $0 \%$ & $\mathbf{4 2 \%}$ & $\mathbf{5 8 \%}$ \\
\hline Generar predicciones e hipótesis & $8 \%$ & $17 \%$ & $58 \%$ & $17 \%$ \\
\hline Estimular el espíritu crítico & $8 \%$ & $8 \%$ & $\mathbf{5 0} \%$ & $\mathbf{3 4 \%}$ \\
\hline Laboratorios virtuales & $\mathbf{5 0 \%}$ & $\mathbf{5 0} \%$ & $0 \%$ & $0 \%$ \\
\hline Simulaciones & $\mathbf{3 4 \%}$ & $\mathbf{4 2 \%}$ & $16 \%$ & $8 \%$ \\
\hline Modelización & $\mathbf{4 2 \%}$ & $\mathbf{4 2 \%}$ & $16 \%$ & $0 \%$ \\
\hline Análisis de datos & $8 \%$ & $34 \%$ & $42 \%$ & $16 \%$ \\
\hline Presentación de datos & $0 \%$ & $16 \%$ & $\mathbf{1 6 \%}$ & $\mathbf{6 8 \%}$ \\
\hline Trabajo cooperativo entre el alumnado & $8 \%$ & $25 \%$ & $\mathbf{5 0} \%$ & $17 \%$ \\
\hline Trabajo cooperativo entre el profesorado & $8 \%$ & $8 \%$ & $\mathbf{4 2 \%}$ & $\mathbf{4 2 \%}$ \\
\hline Trabajo cooperativo entre alumnado y profesorado & $8 \%$ & $25 \%$ & $\mathbf{5 9 \%}$ & $8 \%$
\end{tabular}

Fuente: elaboración propia 
En general, la visión del profesorado acerca de la utilidad del iPad para fomentar la competencia científica a través del desarrollo de las diferentes fases del método científico es bastante buena. Al igual que ocurría con los estudiantes (Tabla 1), los docentes también consideran que las fases de documentación y ordenamiento de la información, así como la presentación de datos son las que más se fomentan con el iPad, mientras que la experimentación virtual a través del uso de laboratorios virtuales y simulaciones las que menos (Tabla 2). Además, según la opinión del profesorado el iPad es una buena herramienta para favorecer el trabajo cooperativo, especialmente entre el profesorado, y que también favorece la autonomía de los alumnos (Tabla 2).

Casi un 92\% del profesorado coincide en que tanto la creatividad como el gusto por lo estético se fomenta considerablemente gracias al uso del iPad. Las actividades que fomentan en mayor grado el desarrollo científico-creativo son: la elaboración y exposición de trabajos y la elaboración de videos. (Gráfico 7).

GRÁFICO 7. Desarrollo científico-creativo a través del iPad en actividades según el profesorado

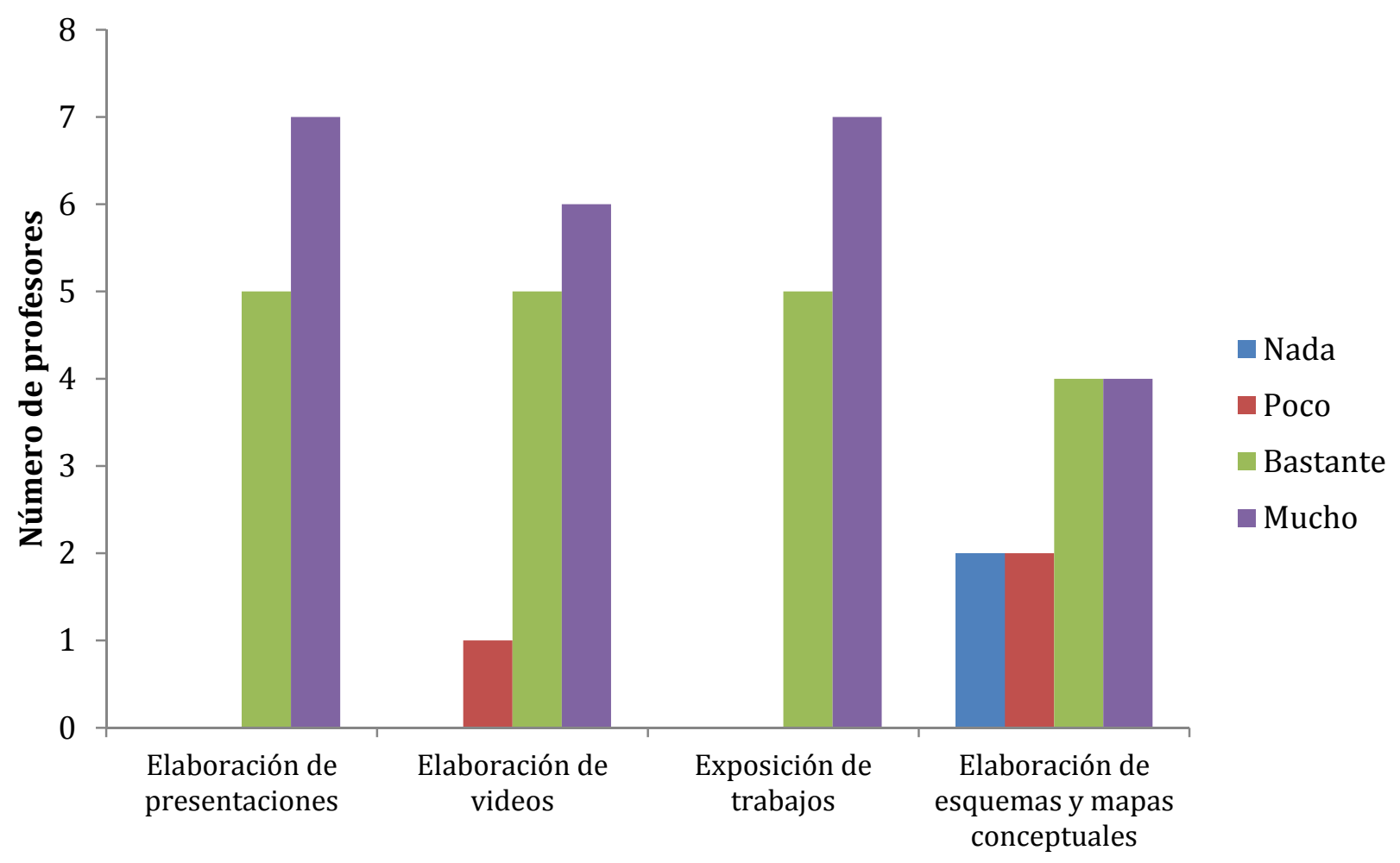

Fuente: elaboración propia

El 100\% de los profesores parece estar de acuerdo en que el iPad ha fomentado su motivación en clase (50\% mucho y $50 \%$ bastante). Sin embargo, cuando se les pregunta acerca del cambio motivacional en los alumnos, aunque la mayoría (75\%) cree que este aumento de la motivación también se ha visto reflejado en los estudiantes, un 25\% considera que el cambio motivacional es escaso en los alumnos.

Pese a todas las ventajas del iPad manifestadas por el profesorado, casi el 92\% afirman que no aprovechaban todos los recursos que les ofrece el dispositivo, argumentando que desconocen muchos de estos recursos y que ha pasado poco tiempo que hace desde su implantación. Consideran, además, que la formación del profesorado en estas tecnologías es esencial. Por otro lado, una de las mayores desventajas del iPad por parte del alumnado es la aparente pérdida de atención que conlleva su uso. El 58\% de los docentes consideran que el iPad perjudica mucho o bastante la atención de los alumnos siendo los principales motivos por los cuales los alumnos se 
distraen tienen relación directa con aplicaciones como el correo electrónico, juegos, videos y las búsquedas en Internet no relacionadas con las clases. No obstante, la calificación global proporcionada por el profesorado a los beneficios del iPad es de 7,5 (Gráfico 4), muy por encima de la de $1^{\circ}$ de Bachillerato que es de un 5,6. Sin embargo, exceptuando este curso, no existen diferencias significativas entre la calificación del profesorado y los cursos de Educación Secundaria Obligatoria. Analizando el gráfico 4, se observa como la percepción del profesorado sobre el beneficio del iPad en el aprendizaje se acerca a la de los cursos inferiores, de hecho la calificación de $1^{\circ}$ de E.S.O. de un 7,2 es muy similar, aumentando la diferencia a medida que avanzan los cursos.

\section{DiSCUSIÓN}

Nuestros resultados muestran que la percepción general sobre el rendimiento didáctico del iPad por parte del alumnado y el profesorado de Educación Secundaria Obligatoria (E.S.O.) y Bachillerato es positiva. El iPad puede ser un excelente mediador para cuestiones específicamente curriculares (Palmero y Rodríguez, 2012), algo que apoyan nuestros resultados, ya que los alumnos los señalan como una de las herramientas preferidas a la hora de estudiar. Cabe destacar el poco uso que hacen los alumnos del libro físico, lo que muy probablemente puede explicarse por el hecho de que posean ese mismo libro en versión digital en sus iPad. Además de la versión digital de los libros de texto, una de las grandes ventajas es la gran variedad de aplicaciones y recursos que ofrecen. Sin embargo, muchas veces se presentan incompatibilidades, de tipo comercial o sistémicas, con otras herramientas como el ordenador (Ayestaran, 2012). Esto apoyaría el hecho de que más del 80\% de los alumnos afirmen que el uso que le dan al iPad fuera del ámbito escolar sea principalmente académico, ya que muchos trabajos que deben realizar en casa solamente pueden hacerlos desde programas únicamente compatibles con iPad. Además, uno de los mayores reclamos para la implementación del iPad en el ámbito educativo es el fomento del trabajo en grupo, las actitudes sociales, el intercambio de ideas, la cooperación y la personalidad (Gil, 2013). Nuestros resultados muestran que la mayoría de alumnos y profesores coinciden en la utilidad del iPad para fomentar estos valores, no sólo entre ellos, sino también entre las diferentes materias del centro. Asimismo, la motivación de docentes y alumnos es otro de los aspectos que a ojos del profesorado se ve aumentada gracias al iPad, como también han sugerido otros estudios (Gil, 2013).

Docentes y estudiantes coinciden en que en las asignaturas relacionadas con las ciencias de la vida (principalmente Biología y Geología) es donde más rendimiento se obtiene del iPad. LaparteCampo (2013) encontró resultados similares, y uno de los principales motivos sería que el contenido de estas asignaturas se presta en gran medida a trabajar con este tipo de dispositivos, como la facilidad para la obtención de información (dibujos y esquemas de modelos biológicos, animaciones y videos de procesos geológicos y biológicos, etc.). De hecho, tanto alumnos como profesores coinciden en que el iPad es de gran utilidad para búsqueda y ordenamiento de la información. En este contexto sería también esperable el impacto del gran potencial que tiene el iPad en las simulaciones y la experimentación virtual tan importante en este tipo de asignaturas (por ejemplo, disecciones virtuales), pero sorprendentemente la gran mayoría de estudiantes y docentes afirman no haber usado simulaciones ni laboratorios virtuales. Uno de los motivos podría ser, por un lado, la inversión en tiempo necesaria para diseñar laboratorios virtuales y, por otro, la falta de resultados empíricos acerca de su uso (García y Ortega, 2007). Además, el centro donde se llevó a cabo este estudio está dotado con varios laboratorios físicos en los que los alumnos realizan prácticas de un modo tradicional, sirviendo el iPad como apoyo para el desarrollo de estos laboratorios y no como un sustituto. No obstante, también hay que considerar que hay prácticas con un gran potencial didáctico que por ciertas limitaciones, como la falta de espacio y tiempo o la peligrosidad, no pueden llevarse a cabo de manera física, siendo en este caso el iPad un sustituto del trabajo experimental. 
Las herramientas TIC que los profesores animan a usar a sus alumnos suelen ser las mismas que los alumnos manejan con cierta soltura (Gómez, Cañas, Gutiérrez y Martín, 2014), lo que hace que las opiniones de docentes y estudiantes sobre los beneficios de su uso normalmente coincidan. No obstante, es de resaltar que en nuestro estudio hemos encontrado que los estudiantes valoran más positivamente el iPad en los primeros cursos de la E.S.O. y menos positivamente en Bachillerato. De hecho, los docentes son los que mayor nota media ponen al iPad (7.5 sobre 10), calificación bastante similar a la de $1^{\circ}$ de E.S.O. Sin embargo, esta calificación decrece con los cursos, llegando a un 5,6 en $1^{\circ}$ de Bachillerato. Esto puede ser debido, por un lado, al desencantamiento del alumnado a medida que el contenido curricular cambia y se va endureciendo, y por otro, que los cursos superiores están más acostumbrados a la metodología tradicional ya que el iPad lleva relativamente poco tiempo implantado en el centro. En esta línea, los resultados sobre los métodos de evaluación preferidos por los alumnos muestran cómo, a medida que avanzan los cursos, el tradicional examen a papel gana la batalla a los novedosos métodos de evaluación basados en cuestionarios y juegos online. Cuando se les preguntó el motivo, muchos alumnos afirmaban que en el papel podían expresarse mejor, desarrollar más las ideas y estar más concentrados, además de poder volver a preguntas anteriores si era necesario. Además, para los alumnos el nivel de distracción debido al iPad también iría e aumento a medida que avanzan los cursos. Esto podría deberse a que con cada curso, el alumno va ampliando los intereses no relacionados con el ámbito académico, de manera que son más conscientes del potencial de distracción y del perjuicio que conlleva sobre su aprendizaje.

Es muy llamativo que más del $90 \%$ de los docentes coinciden en apuntar que no hacen un aprovechamiento completo del iPad, señalando como principales motivos el poco tiempo desde la implantación del dispositivo en el centro y el desconocimiento de muchos de sus recursos. Esta puede ser también una razón que explique su escaso uso como herramienta para simulaciones y experimentación virtual. De hecho, nuestros resultados muestran que el mayor rendimiento del iPad obtenido por los profesores está directamente relacionado con la elaboración de apuntes y la búsqueda en Internet (algo que puede también hacerse en un ordenador) y no con otras posibilidades y herramientas innovadoras que ofrece el iPad. Esto pone de manifiesto la importancia de la formación inicial y continua del profesorado en el uso de las nuevas tecnologías para obtener un mejor rendimiento de ellas (Gil, 2013). Además, la formación del profesorado no es sólo importante para conocer todas las posibilidades que ofrecen los iPad o tabletas, sino que una dimensión importante es saber trabajar en clase con alumnos que utilizan estas metodologías. Esto puede reducir el impacto de estos dispositivos en la distracción por parte de los alumnos. En este sentido, tanto alumnos como profesores muestran una variabilidad de opiniones respecto de esta cuestión, lo que apunta a que hay una gran diversidad en el alumnado y de aulas en cuanto a la capacidad de estos dispositivos para distraer a los alumnos. La metodología docente, por tanto, puede ser un eje importante a trabajar en la formación del profesorado en estas nuevas tecnologías. Nuestros resultados sugieren que las TIC en general, y el iPad en particular, no pueden considerarse por sí solas como un signo de elevada calidad educativa. Su uso requiere de una formación adecuada del profesorado y de una metodología de trabajo específica en el aula que son los elementos que hacen desarrollar todo el potencial de estas herramientas en el contexto de la enseñanza-aprendizaje de las ciencias. En función de cómo se trabajen estos aspectos, el iPad puede ser una fuente inagotable de información y motivación, o un elemento capaz de distraer y entorpecer la dinámica del aula (Blanch, 2013). Además, una docencia de calidad quizá resida en la combinación de antiguas y nuevas tecnologías y metodologías. Los dispositivos tecnológicos no pueden sustituir a determinadas las prácticas y herramientas didácticas de larga tradición, como el trabajo experimental directo (Vásquez-Rodríguez, 2015). Es innegable el gran potencial para la transformación de la educación científica que encierra cada uno de los dispositivos tecnológicos. Sin embargo, la significación que se alcance con estas tecnologías dependerá de la capacidad de la 
comunidad educativa de integrarlos adecuadamente en la dinámica de las clases y de relacionar su uso con el resto de variables curriculares (ver Cabero, 2007).

\section{Referencias}

Ancira, A. y Gutiérrez, F. (2011). Integración y apropiación de las TIC en los profesores y los alumnos de educación media superior. Revista de Innovación Educativa, 3(1), 1-10.

Ariza, M. R. y Quesada, A. (2014). Nuevas tecnologías y aprendizaje significativo de las ciencias. Enseñanza de las Ciencias, 32 (1), 101-115. DOI: 10.5565/rev/ensciencias.433

Aunión, J. A. (2009). La era del profesor desorientado. Diario El País [en línea], 32-33. Recuperado de http://www.stecyl.es/prensa/090718_profesor_desorientado.pdf.

Blanch, J. M. (2013). El trabajo académico digital como factor de riesgo psicosocial. Usos y abusos de las TIC en la educación superior. En L. Bianchetti y T. M. Hetkowski (Eds.), Dossiê: Novas tecnologias, formação docente e pósgraduação. Monográfico. Educação em Perspectiva (pp. 511-532).

Cabero, J. (2007). Las necesidades de las TIC en el ámbito educativo: oportunidades, riesgos y necesidades. Tecnología y comunicación educativas, Año 21 (45). Recuperado de http://investigacion.ilce.edu.mx/tyce/45/articulo1.pdf.

Carneiro, R., Toscano, J. C., y Díaz, T. (Coords.) (2009). Los desafíos de las TIC para el cambio educativo. Madrid: OEI, Fundación Santillana.

Galaz, C. M., Weil, C. G. (2014). Concepciones del profesorado universitario acerca de la ciencia y su aprendizaje y cómo abordan la promoción de competencias científicas en la formación de futuros profesores de Biología. Enseñanza de las ciencias, 32(1), 51-81. DOI: $10.5565 /$ rev/ensciencias.852

García, G. A., y Rey, R. (2012). Las apps en el aula del siglo XXI. Comunicación y Pedagogía. Recuperado de http://www.centrocp.com/las-apps-enel-aula-del-siglo-xxi/.

García, M. L., y Ortega, J. G. M. (2007). Las TIC en la enseñanza de la Biología en la educación secundaria: los laboratorios virtuales. Revista electrónica de Enseñanza de las Ciencias, 6(3), 562-576. Recuperado de http://reec.uvigo.es/volumenes/volumen6/ART5_Vol6_N3.pdf

García-Vera, A. B. (2004). Calidad de la educación en la sociedad de la información. Revista Complutense de Educación, 15(2), 509-520.

Gil, S. J. J. (2013). iPad y motivación. Revista Didáctica, Innovación y Multimedia, 26. Recuperado de http://dim.pangea.org/revistaDIM26/revista260Clipadymotivacion.htm.

Gómez, M. A., Cañas, A. M., Gutiérrez, M. S., y Martín, M. J. (2014). Ordenadores en el aula: ¿estamos preparados los profesores? Enseñanza de las Ciencias, 32(2), 239-250. DOI: $\underline{10.5565 / \text { rev/ensciencias.939 }}$

Gómez-Crespo, M. A., Pozo, J. I., y Gutiérrez-Julián, M. S. (2004). Enseñando a comprender la naturaleza de la materia: el diálogo entre la química y nuestros sentidos. Educación Química, 15(3), 198-209.

González, V. O. (2011). Los modelos 1:1 en educación: Prácticas internacionales, evidencia comparada e implicaciones políticas. Revista Iberoamericana de Educación, 56, 113-134.

Gordillo, M., Tedesco, J., López, J., Acevedo, J., Echeverria, J., y Osorio, C. (2009). Educación Ciencia Tecnología y Sociedad. Documentos de Trabajo N³. Madrid: OEI.

Gracia, M., Vigo, M., Marcó, M., y Fernández, M. (2002). Problemas conductuales relacionados con el uso de Internet: Un estudio exploratorio. Anales de la Psicología, 18(2), 273-292.

Guitert, M., Romeu, T., y Perez-Mateo, M. (2007). Competencias TIC y trabajo en equipo en entornos virtuales. Revista de Universidad y Sociedad del Conocimiento, 4(1), 1-12.

Laparte-Campo, M. (2013). Instrumento de estudio del uso de las TIC en la práctica docente de Educación Secundaria (Proyecto fin de máster). Máster universitario en formación del profesorado de educación secundaria. Universidad Internacional de La Rioja, España. 
Lowy, E. (1999). Utilización de Internet para la enseñanza de las ciencias. Alambique. Didáctica de las Ciencias experimentales, 19, 65-72.

Marcelo, C., y Estebaranz, A. (1999). Cultura escolar y cultura profesional: los dilemas del cambio. Revista Educar, 24, 47-147. DOI: 10.5565/rev/educar.334

Marés, L. (2012). Tablets en Educación: Oportunidades y desafíos en políticas uno a uno. OEI. Oficina Regional Buenos Aires. Argentina. Recuperado de http://www.oei.es/70cd/Tabletseneducacion.pdf.

Martínez, C. J. A. (2015). Lo cotidiano (entre usos y resistencias) de las tic en un aula de la educación secundaria obligatoria. Revista de Antropología Experimental, 15, 567-580. DOI: 10.17561/rae.v0i15.2633

Nieves, J. M. (2013). El uso de tablets mejora la comprensión de los estudiantes de ciencias. Diario $A B C$ [en línea] Recuperado de http://www.abc.es/tecnologia/moviles-tabletas/20131209/abcitablets-ipad-estudiantes-201312090937.html.

Palmero, J. R., y Rodríguez, J. S. (2012). Expectativas de los centros educativos ante los proyectos de integración de la TIC en las aulas. Revista de Educación, 357, 587-613. DOI: 104438/1988-592X-RE-2010-357-118

Pedrajas, P. A. (2005). Aplicaciones de las tecnologías de la información y de la comunicación en la educación científica. Primera parte: funciones y recursos. Revista Eureka sobre Enseñanza y Divulgación de las Ciencias, 2(1), 2-18. DOI: 10.25267/Rev_Eureka_ensen_divulg_cienc. 2005.v2.i1.02

Sigales, C., Mominó, J. M., Meneses, J., y Badía, A. (2008). La integración de Internet en la educación escolar española: situación actual y perspectivas de futuro. Madrid: Ariel.

Tallone, A. E. (2011). Las transformaciones de la autoridad docente, en busca de una nueva legitimidad. OEI Buenos Aires, Argentina. Revista de Educación, 2011, 115-135.

Vásquez-Rodríguez, F. (2015). La necesidad de orientar el uso didáctico de las nuevas tecnologías. Ruta Maestra, 12-15. Recuperado de http://www.santillana.com.co/rutamaestra/edicion1/pdf/2.pdf.

\section{CÓMO CITAR ESTE ARTÍCULO}

Ruiz Santiago, A., Muñoz Muñoz, A., Valladolid Carriazo, M. D. y Peña Martínez, J. (2019). El iPad en la Educación científica de estudiantes de Secundaria y Bachillerato. Didáctica de las ciencias experimentales y sociales, 36, 97-114. DOI: 10.7203/DCES.36.12661. 


\section{Anexo I: Cuestionarios}

\section{CUESTIONARIO PARA LOS ALUMNOS}

1. ¿Con cuál de las siguientes herramientas sueles estudiar?
*IPad
*Libro físico
*Apuntes
*Ordenador

2. Fuera de clase ¿Cuánto tiempo sueles estar usando el IPad?

*Menos de 1 h al día *Aproximadamente 1 h al día

*Entre 1 y 2 h al día *Más de 3 h al día

3. Fuera de clase $¿$ Con qué frecuencia sueles usar el IPad para realizar las siguientes actividades? [0:nada, 1:poco, 2:bastante, 3:mucho]
*Estudiar
*Ver series/películas
*Enviar/recibir correos *Navegar por internet *Jugar
*Elaborar trabajos
*Leer

4. ¿Cuánto usas el IPad para aprender en clase en las siguientes asignaturas? [0:nada, 1:poco, 2:bastante, 3:mucho y NP no procede]
*Matemáticas
*Física y Química
*Biología y Geología
*Biología
*Tecnología *Ciencias naturales *Anatomía

5. ¿Te sirve de ayuda el IPad para la búsqueda de información sobre los contenidos de las diferentes asignaturas? [0:nada, 1:poco, 2:bastante, 3:mucho]

6. ¿Te sirve de ayuda el IPad para elaborar trabajos con tus compañeros? [0:nada, 1:poco, 2:bastante, 3:mucho]

7. ¿Te facilita el IPad el agrupamiento y la ordenación de la información que encuentras? [0:nada, 1:poco, 2:bastante, 3:mucho]

8. ¿Has usado alguna vez simulaciones o juegos matemáticos? [0:nada, 1:poco, 2:bastante, 3:mucho]

9. ¿Has usado alguna vez alguna aplicación o alguna página de laboratorios virtuales? [0:nada, 1:poco, 2:bastante, 3:mucho]

10. ¿Cuáles de los siguientes métodos de evaluación te gusta más?

*Socrative *Kahoot *Eamen en papel

11. ¿Usas el IPad para el análisis y representación gráfica de datos con herramientas similares a Excel? [0:nada, 1:poco, 2:bastante, 3:mucho]

12. ¿Te es más fácil trabajar por ti mismo y sin ayuda gracias al IPad? [0:nada, 1:poco, 2:bastante, 3:mucho]

13. ¿Te permite el IPad desarrollar trabajos más creativos y con un mejor resultado estético? [0:nada, 1:poco, 2:bastante, 3:mucho]

14. ¿Te distraes más en clase o estudiando cuando usas el IPad? [0:nada, 1:poco, 2:bastante, 3:mucho]

15. ¿Sueles hacer alguna de estas acciones con el iPad en clase? [0:nada, 1:poco, 2:bastante, 3:mucho]

*Navegar por internet *Jugar

*Escuchar música *Enviar/recibir correos *Ver videos

16. Valora del 1 al 10 lo beneficios del iPad para tu aprendizaje [ 1 nada beneficioso, 10 muy beneficioso] 
CUESTIONARIO PARA LOS DOCENTES

1. ¿En qué asignaturas sacas un mayor partido al IPad? [0:nada, 1:poco, 2:bastante, 3:mucho]

*Matemáticas

*Física y Química

*Tecnología

*Ciencias naturales *Biología y Geología

*Anatomía

2. ¿Qué tiempo de clase haces uso del IPad para trabajar los contenidos? $0=$ nada, $1=$ menos de $1 / 2$ de sesión, $2=$ $1 / 2$ de sesión, $3=$ más de $1 / 2$ de sesión y NP

*Matemáticas *Física y Química

*Ciencias naturales *Biología y Geología *Anatomía

3. ¿Qué tipo de uso sueles hacer del IPad como docente?

*Elaboración de apuntes *Elaboración y exposición de presentaciones *Elaboración de juegos

*Elaboración de videos * *Proyección de videos

*Elaboración de esquemas y mapas conceptuales

*Búsqueda en internet desde clase

*Elaboración de exámenes y

cuestionarios

4. ¿Qué condiciona más el uso del IPad en clase?

*El contenido de la asignatura $\quad$ *El número de horas de la asignatura $\quad$ *La cantidad de temario *Otros:

5. Valora la utilidad del IPad para el desarrollo de las siguientes actividades por parte del profesor: [0:nada, 1:poco, 2:bastante, 3:mucho]

*Indagar, recoger datos y documentarse *Agrupar, ordenar, contrastar información

*Generar predicciones e hipótesis *Estimular el espíritu crítico

6. ¿Consideras el IPad una buena herramienta para la experimentación virtual? [0:nada, 1:poco, 2:bastante, 3:mucho]

7. ¿Con qué frecuencia sueles usar los siguientes recursos en clase a través del IPad? [0:nada, 1:poco, 2:bastante, 3:mucho]

*Laboratorios virtuales *Simulaciones *Herramientas de modelización

*Herramientas de análisis de datos *Herramientas de presentación de datos

8. ¿Crees que la experimentación virtual con el uso del IPad ayuda a la obtención de conclusiones por parte del alumnado? [0:nada, 1:poco, 2:bastante, 3:mucho]

9. ¿Consideras que el IPad fomenta el desarrollo de trabajo cooperativo? [0:nada, 1:poco, 2:bastante, 3:mucho]

*Entre el alumnado *Entre el profesorado *Entre el alumnado y el profesorado

10. ¿El uso del IPad en clase fomenta el trabajo autónomo por parte del alumnado? [0:nada, 1:poco, 2:bastante, 3:mucho]

11. ¿Es el IPad una buena herramienta para el desarrollo científico-creativo del alumno? [0:nada, 1:poco, 2:bastante, 3:mucho]

12. ¿Crees que estás aprovechando al $100 \%$ todos los recursos del IPad en tus clases?

13. En caso contrario, indica las limitaciones que limitan el aprovechamiento [0:nada, 1:poco, 2:bastante, 3:mucho]

*Desconocimiento de recursos *Poco tiempo desde su implantación

*Por la predisposición del alumnado $\quad$ *Por la predisposición del profesorado $\quad$ *Otros:

14. ¿Crees que la renovación metodológica con el uso del IPad ha supuesto un cambio motivacional? [0:nada, 1:poco, 2:bastante, 3:mucho]

*Por parte del alumnado *Por parte del profesorado

15. ¿Crees que el IPad perjudica a la capacidad atencional del alumno? [0:nada, 1:poco, 2:bastante, 3:mucho]

16. ¿ Usa indebidamente el alumnado el IPad para estas actividades en horario de clase? [0:nada, 1:poco, 2:bastante, 3:mucho]

*Correo electrónico *Juegos *Búsquedas internet no relacionadas con la clase

*Escuchar música sin permiso $\quad$ *Visionar videos no relacionados con la clase

17. Valora del 1 al 10 los beneficios del IPad para el aprendizaje de los alumnos 